\title{
Full-Filling Subcarrier Power Allocation in OFDMA-Based Cognitive Radio Systems
}

\author{
Tuan Le Van ${ }^{1}$, Hieu Dinh $\mathrm{Chi}^{2}$, Kinh Nguyen Viet ${ }^{1}$, Hieu Nguyen Thanh ${ }^{3}$ \\ ${ }^{1}$ University of Engineering and Technology, Hanoi, Viet Nam; ${ }^{2}$ Authority of Radio Frequency Management, Hanoi, Viet Nam; \\ ${ }^{3}$ School of Electrical and Electronic Engineering, Nanyang Technological University, Singapore City, Singapore. \\ Email: tuanlv@rfd.gov.vn
}

Received October $28^{\text {th }}, 2013$; revised November $30^{\text {th }}, 2013$; accepted December $19^{\text {th }}, 2013$

Copyright (C) 2014 Tuan Le Van et al. This is an open access article distributed under the Creative Commons Attribution License, which permits unrestricted use, distribution, and reproduction in any medium, provided the original work is properly cited. In accordance of the Creative Commons Attribution License all Copyrights (C) 2014 are reserved for SCIRP and the owner of the intellectual property Tuan Le Van et al. All Copyright (C) 2014 are guarded by law and by SCIRP as a guardian.

\section{ABSTRACT}

When implementing an appropriate windowing, the interference from a Cognitive Radio (CR) system to licensed systems (primary users) will be significantly reduced. Consequently, power allocated to subcarriers can be increased, especially subcarriers having far spectral distance to primary user bands can be allocated full of its maximum possible power. In this paper, we propose a new class of sub-optimal subcarrier power allocation algorithm that significantly reduces complexity of OFDMA-based CR systems. Two sub-optimal proposals, called Pre-set Filling Range (PFR) and Maximum Filling Range (MFR) are studied. Investigations show that this new power allocating algorithm allows CR systems obtain high throughput while retaining low complexity.

\section{KEYWORDS}

\section{Cognitive Radio; Windowing; Power Allocation; Full Filling}

\section{Introduction}

Today, rapid growth of data demands requires solutions, especially higher spectrum efficiency solution, to satisfy the bandwidth hunger. Thanks to its ability of dynamically exploiting unused spectrum, OFDM-based Cognitive Radio (CR) has been considered widely as a solution to ameliorate spectrum efficiency. However, because of the use of unused spectrum of licensed users (or primary user-PU), CR and primary users often exist in side by side bands, thus interference may cause from CR to PU system and vice versa. As presented in [1-4], being based on OFDMA technology, capacity of a CR system depends not only on powers allocated to each of its subcarrier but also on interference level caused from PU users to CR user's band. It was further explained in [1], [5]. This mutual interference is due to the non-orthogonality of OFDM signals. This mutual interference leads to the reduced performance of both PU and CR networks. In principle, higher powers allocated to its subcarriers, higher capacity CR could obtain, but higher level of interference could cause PU. With the presence of mutual interference, amounts of power to be allocated to each CR's subcarriers must be assessed in a way to ensure the total interference caused from all subcarriers to PU's bands not exceed a pre-defined threshold to protect licensed primary user. Therefore, improving transmit capacity while keeping interference to licensed user at an acceptable level is a challenge which needs to be studied in the implementation of CR system.

So far, a number of studies have been carried out to find solutions to reduce mutual interference with aims to increase subcarrier power and hence CR capacity. A side-lobe suppression mechanism has been proposed in [6] in order to minimize the adjacent channel interference. A max-min algorithm was proposed in [7] for subchannel, bit and power allocation in a multiuser OFDMbased CR system which shows a better system performance compared with the case that uses guard-bands to protect the active PU users. In [5], the mutual interference problem was studied under the condition that CR system uses OFDM modulation. In [1], two suboptimal schemes have been also proposed in which different step sizes were used in allocating power to each sub-carrier. 
In [2], several sub-optimal schemes (scheme A and B) were suggested to reduce numerical calculation steps. The effect of a subcarrier nulling and performance of classical power loading algorithm (water filling, uniform power allocation) has been investigated.

In [8], the authors proposed using windowing to reduce the interference from CR to PU. Results of this study show that, as can be seen from Figure 1, only a few subcarriers nearby the PU are affected and their allocated power need to be adjusted carefully, other CR subcarriers can be allocated full power as they introduce negligible interference to PU system. This does not happen in case when no windowing is introduced to CR in which almost CR subcarriers are not allocated the full power (see Figure 2). In terms of system capacity, employing windowing significantly improved the capacity.

Based on the results of study in [8], this paper proposes a new class of power allocation algorithm, namely

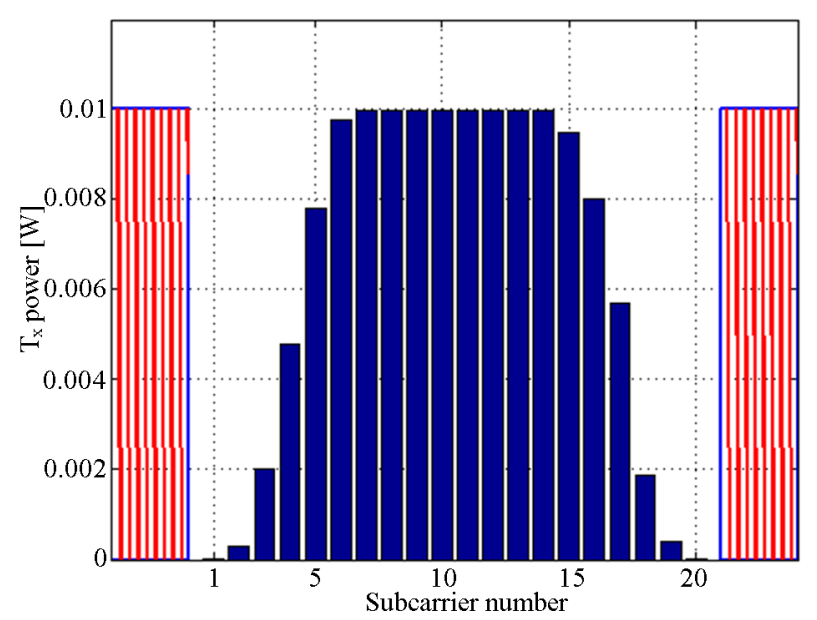

Figure 1. Optimal CR's power allocation (with Tukey window, $I_{t h}=5 \sigma^{2}$ ).

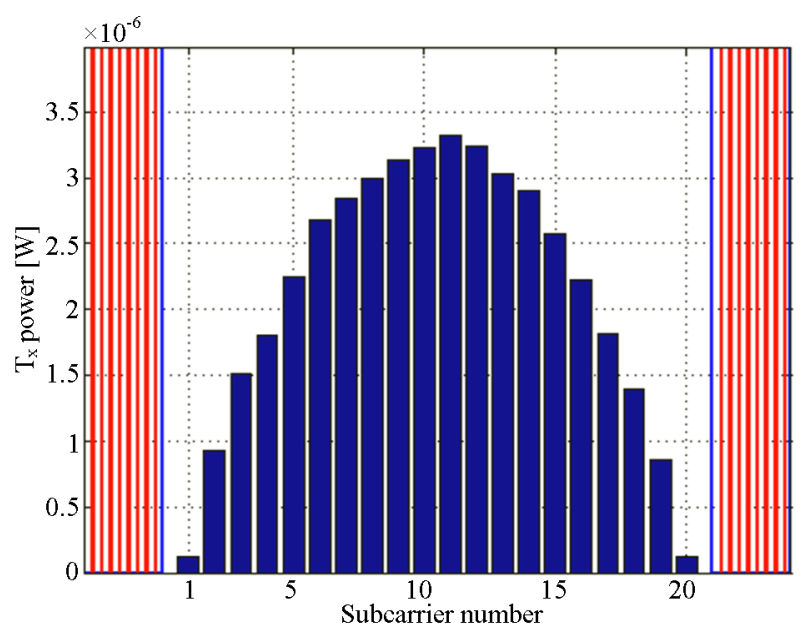

Figure 2. Optimal CR's power allocation (without Tukey window, $I_{t h}=5 \sigma^{2}$ ). full-filling subcarrier power allocation and assesses impact of this new algorithm to system capacity and computational complexity. Two sub-optimum proposals, called Pre-set Filling Range (PFR) and Maximum Filling Range (MFR) are studied. The central idea of those proposals is allocating full power to sub-carriers that have negligible interference to PU. Investigations show that this new power allocating algorithm allows CR systems obtain high throughput while retaining low complexity.

\section{System Model}

In an OFDM-based CR system operating with presence of PU users, the maximum transmission capacity can be expressed mathematically as [1]:

$$
C=\max \sum_{i=1}^{N} \Delta f \log _{2}\left(1+\frac{\left|h_{i}^{s s}\right|^{2} P_{i}}{\sigma^{2}+\sum_{l=1}^{L} J_{i}^{(l)}}\right)
$$

subject to:

$$
\sum_{i=1}^{L} \sum_{i=1}^{N} I_{i}^{l}\left(d_{i l}, P_{i}\right) \leq I_{t h}
$$

and

$$
P_{i} \geq 0 \quad \forall i=1,2, \cdots, N
$$

where $C$ denotes the transmission capacity of the $C R$ user, $N$ denotes the total number of OFDM subcarriers, $I_{\text {th }}$ denotes the interference threshold can be acceptable by l-th PU band and is a finite deterministic value, $\sigma^{2}$ is the Additive White Gaussian noisk; $f$ is the spectrum bandwidth of a subcarrier, $h_{i}^{\text {ss }}$ is the fading gain, $J_{i}^{(h)}$ is the interference from PU to $i_{t h}$ OFDM subcarrier, $I_{i}^{l}$ is the interference from $i_{t h}$ OFDM subcarrier to PU bands, $P_{i}$ is the transmission power of $i_{t h}$ OFDM subcarrier.

\subsection{Interference from CR to PU}

From Equations (1) and (2), we can see that the CR transmission capacity is affected by the acceptable interference $I_{t h}$ that $P U$ can tolerate.

Assuming signal is an ideal Nyquist pulse, then $i_{\text {th }}$ subcarrier's power spectrum density in $C R$ system can be expressed as [1]:

$$
\phi_{i}(f)=P_{i} T_{s}\left(\frac{\sin \pi f T_{s}}{\pi f T_{s}}\right)^{2}
$$

where $P_{i}$ is the transmission power of $i_{t h}$ OFDM subcarrier, $T_{s}$ is the symbol duration. Interference from subcarrier to PU's bands is integration of this subcarrier spectrum density across the $l_{t h}$ PU band. This interference can be expressed as [1]:

$$
I_{i}^{(l)}\left(d_{i l}, P_{i}\right)=\left|h_{i}^{s p}\right|^{2} P_{i} T_{s} \int_{d_{i l}-\frac{B_{l}}{2}}^{d_{i l}+\frac{B_{l}}{2}}\left(\frac{\sin \pi f T_{s}}{\pi f T_{s}}\right)^{2}
$$


where $d_{i l}$ is the spectrum distance between CR's $i_{t h}$ subcarrier and PU band, $B_{l}$ is bandwidth of $l_{t h} \mathrm{PU}$.

With window at the output, the PSD of CR will be shaped. We will consider a more realistic case at the next section.

\subsection{Interference from $P U$ Users to CR Users}

Power spectrum density of the PU signal after M-fast Fourier transform (FFT) can be expressed as [2]:

$$
E\left\{I_{N}(\omega)\right\}=\frac{1}{2 \pi M} \int_{-\pi}^{\pi} \phi_{P U}\left(\mathrm{e}^{\mathrm{j} \omega}\right)\left(\frac{\sin (\omega-\psi) M / 2}{\sin (\omega-\psi) / 2}\right)^{2}
$$

where $\omega$ is the frequency normalized to the sampling frequency, $\Phi_{P U}$ is power spectrum density of PU signal.

Interference caused by $l_{\text {th }}$ PU signal to the $i_{\text {th }}$ CR's sub-carrier is expressed in [1]:

$$
J_{i}^{(l)}\left(d_{i l}, P_{i}\right)=\left|h_{i}^{s p}\right|^{2} P_{i} T_{s} \int_{d_{i l}-B_{l} / 2}^{d_{i l}+B_{l} / 2} E\left\{I_{N}(\omega)\right\} \mathrm{d} \omega
$$

\subsection{Impact of Windowing on Interference}

The system capacity could be improved by decreasing interference from CR to PU. It could be achieved by windowing transmitting signal of OFDM symbols that sharpens out-of-band subcarrier spectrum which goes down smoothly and quickly. In WLAN system, raisedcosine window is commonly used [9]. This window is defined in time domain as in Equation (10), and associated Fourier transform can be shown as in Equation (11) as in [10].

In case of windowing, the received signal $x(k)$ is windowed in the time domain by window function $w(k)$ result in:

$$
\hat{X}(k)=x(k) w(k)
$$

and the Fourier transform $\hat{X}\left(\mathrm{e}^{j \omega}\right)$ of $\hat{x}(k)$ is a convolution of the Fourier transform $X\left(\mathrm{e}^{j \omega}\right)$ and $W\left(\mathrm{e}^{j \omega}\right)$ of these respective time signals $x(k)$ and $w(k)$

$$
\hat{X}\left(\mathrm{e}^{j \omega}\right)=\frac{1}{2 \pi} \int_{-\pi}^{\pi} X\left(\mathrm{e}^{j \omega}\right) W\left(\mathrm{e}^{j(\omega-\psi)}\right) \mathrm{d} \psi
$$

$$
G(f)=\frac{\pi^{2}\left(\sin \left(\frac{T \omega}{2}(1-a)\right)+\sin \left(\frac{T \omega}{2}\right)\right)}{\omega\left(\pi^{2}-\omega^{2} a^{2}\left(\frac{T}{2}\right)^{2}\right)}
$$

\subsection{PU's Signal Model}

In theory, cognitive radio can work in any frequency bands, but in practical broadcasting UHF band is focused as a high potential to host the first secondary wireless networks. TV UHF band has been chosen thank to its suitable propagation characteristics for wireless systems like CRS and to a fact that in this band, close-by terrestrial transmitting TV stations normally use different frequencies to avoid interference between them and this opens "white space" chance for secondary systems to use.

Based on this fact, in this study we assume PU's signal is a DVB-T signal for interference assessment between CR and PU.

\section{Full-Filling Algorithm}

As mentioned in Section I, when implementing windowing, only subcarriers nearby PU users create significant amount of interference to CR, for subcarriers having far spectral distance, interference are negligible. Therefore, those subcarriers could be loaded with maximum power level. Based on this observation, we have developed a new sub-optimum algorithm class, namely full-filling, as follow:

The first proposed algorithm, called Max Filling Range (MFR), is:

\section{FOR each frame transmission}

- Collect channel information;

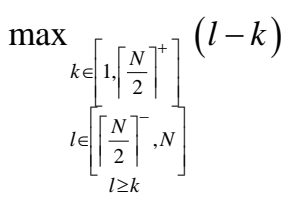

subject to

for each $l: \sum_{i=k}^{l} I_{i}^{l} \leq I_{t h}$

$$
f(n)= \begin{cases}\frac{1}{2}\left(1+\cos \left(\frac{2 \pi}{a T}\left[t-\frac{T}{2}(1-a)\right]\right)\right), & \frac{T}{2}(1-a) \leq t \leq \frac{T}{2}, 0 \leq a \leq 1 \\ 1, & -\frac{T}{2}(1-a) \leq t \leq \frac{T}{2}(1-a) \\ \frac{1}{2}\left(1-\cos \left(\frac{2 \pi}{a T}\left[t+\frac{T}{2}\right]\right)\right), & -\frac{T}{2} \leq t \leq-\frac{T}{2}(1-a)\end{cases}
$$


Set $K \rightarrow k \& L \rightarrow l$

$$
C_{\text {MFR }}=\sum_{i=K}^{L} \Delta f \log _{2}\left(1+\frac{\left|h_{i}^{s s}\right|^{2} P_{\max }}{\sigma^{2}+\sum_{l=1}^{L} J_{i}^{l}}\right)
$$

IF no MFR solution: solve (1).

The merit of MFR algorithm is its simplicity. The algorithm is very fast to converge, there for it is suitable for power saving and low computation capacity CR's applications.

For a better performance while still keeping low complexity, we propose the second algorithm, called Pre-set Filling Range (PFR), as following:

FOR each frame transmission

STEP 1)

- Collect channel information;

- Set the filling range: $K, L$;

- Pre-calculate:

$$
C_{F F}=\sum_{i=K}^{L} \Delta f \log _{2}\left(1+\frac{\left|h_{i}^{s s}\right|^{2} P_{i}}{\sigma^{2}+\sum_{l=1}^{L} J_{i}^{l}}\right)
$$

STEP 2)

- Construct the sub-optimization problem and solve:

$$
C=\sum_{\substack{i=1 \\ i \neq[K, \cdots, M]}}^{N} \Delta f \log _{2}\left(1+\frac{\left|h_{i}^{s s}\right|^{2} P_{i}}{\sigma^{2}+\sum_{l=1}^{L} J_{i}^{l}}\right)+C_{F F}
$$

Subject to

$$
\begin{aligned}
& \bigvee_{l=1}^{L}\left(\sum_{\substack{i=1 \\
i \neq[K, \cdots, M]}}^{N} I_{i}^{l}\left(d_{i l}, P_{i}\right)+\sum_{i=K}^{M} I_{i}^{l}\left(d_{i l}, P_{\max }\right)\right) \\
& \leq I_{\text {th }} 0 \leq P_{i} \leq P_{\max } \quad \forall i=\{1, \cdots, N\} \backslash\{K, \cdots, M\}
\end{aligned}
$$

RETURN Step 1) for next frame transmission

In comparison with optimal solution, PFR takes a different approach. It allocates lower power to nearby subcarriers in exchange for higher power for far away subcarriers.

The MFR and PFR algorithms are expressed as in Figures 3 and 4:

Normally, in practical frequency assignment, two TV stations are not assigned frequency channels adjacent to each other. The void frequency will serve as gap-band to avoid interference. When the two TV channels are with the same bandwidth, $B_{1}=B_{2}$, then the power allocated to CR's sub-carriers will have the symmetrical form.

\section{Numerical Results}

\subsection{Simulation Parameters}

For comparison purpose, a numerical simulator is devel-

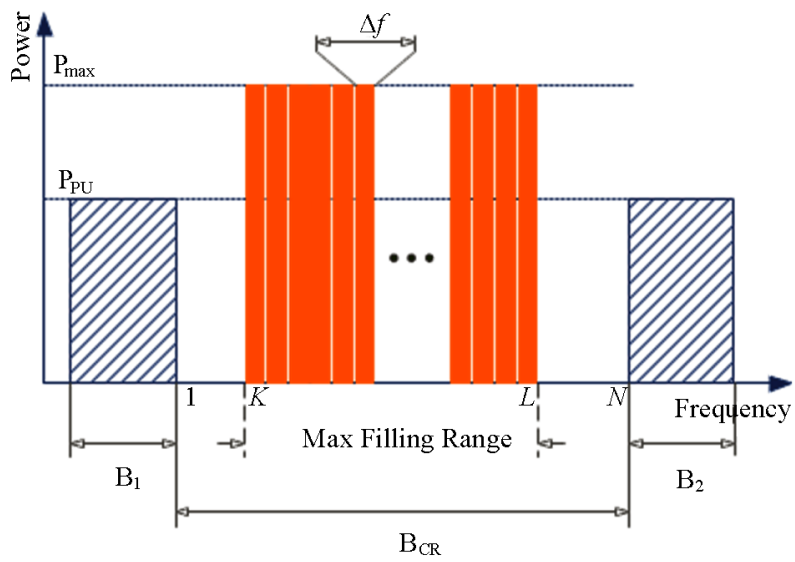

DV Primary User Cognitive User

Figure 3. MFR algorithm.

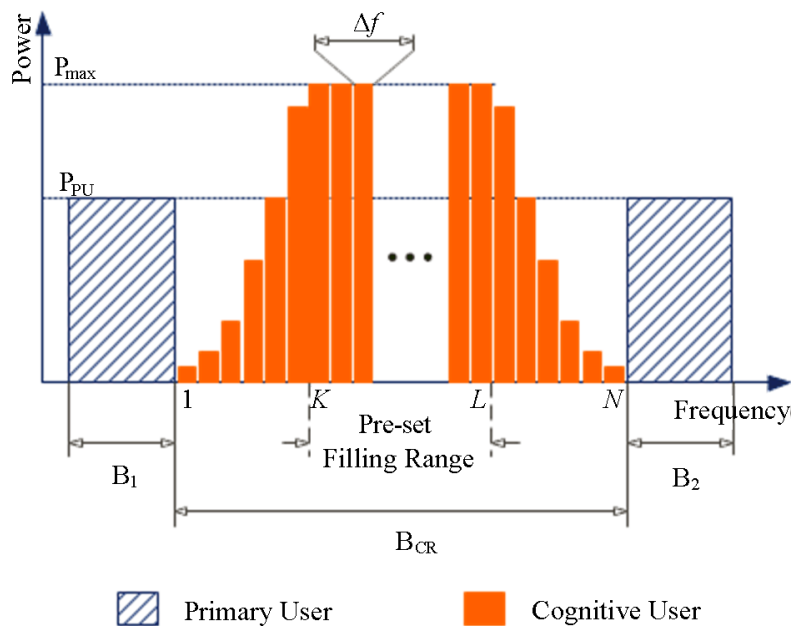

Figure 4. PFR algorithm.

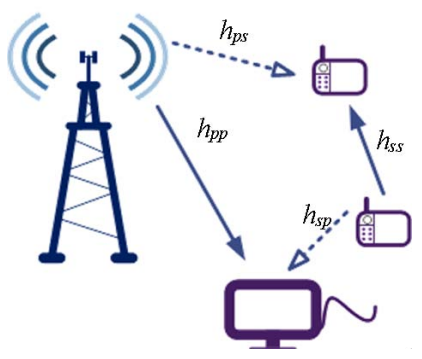

Figure 5. CR's working model.

oped in Matlab. The simulation results are presented in this section; we assume that CR system has 20 subcarriers to operate in $2 \mathrm{PU}$ systems. We assume the value of $T_{s}$ to be $4 \mu$ seconds and $\Delta f$ has been assigned the value of $0.3125 \mathrm{MHz} \mathrm{B}_{1}, \mathrm{~B}_{2}$ varied as $k \Delta f$. The value of $\sigma^{2}$ is assumed to be $10^{-3}$. The value of $\mathrm{P}_{\mathrm{PU}}$ is assumed to be 10 $\mathrm{mW} . P_{C R}$ is calculated at each transmission and is assumed to be no larger than $P_{P U}$.

The working model of CR is demonstrated in Figure 5 
with difference channel gain, communication and interference paths.

Regarding to channel, the channel gains $h_{i}^{s p}, h_{l}^{s p}$, and $h_{l}^{p s}$ are assumed to be Rayleigh fading. Complete channel information is assumed to be known at CR. We consider worst case scenario: the simulator assumes that $\mathrm{CR}$ and PU systems are always transmit data. This approach is reflected in the channel setting as following: $h^{\text {ss }}$ $=5 \mathrm{~dB}, h^{p s}=h^{p p}=10 \mathrm{~dB} ; h^{s p}=15 \mathrm{~dB}$.

Regarding to PU's signal, in this paper, we assume that the transmitting spectrum mask of PU signal is conformable with emission mask defined in the ITU-R Recommendation SM.1541-4 [11]. The Figure 6 is spectrum limit mask for 8MHz DVB-T system.

Regarding to CR's signal, based on the previous study in [8] we apply the Tukey window to CR system. The normalized PSD of Tukey window is demonstrated in Figure 7 . With the introduction of window, the interference from $\mathrm{CR}$ to $\mathrm{PU}$ depends significantly on the roll-off factor of Tukey window.

The values of break points of this mask are in the Table 1:

The CR transmission speed will much depend on the tolerable level of PU against interference that is from CR. As in [8], it is reasonable that the achievable throughput of CR increases in accordance with that tolerable level. The roll-off factor plays a critical role in the maximum achievable throughput of CR system. Throughput increases as the roll-off factor becomes more stringent. Simulation in this paper is conducted with roll-off factor of 0.3 .

The introduction on Tukey window has significantly reduced the interference from $\mathrm{CR}$ to $\mathrm{PU}$ as seen in Figures 1 and 2. Do notice at the difference in y-axis scale of transmission power. Without Tukey window, CR can only transmit with much lower power than that of the Tukey window case. Beside its main objective to reduce interference, Tukey window also opens up possibilities for new suboptimal solutions.

\subsection{Simulation Analysis}

Although PFR algorithm is a sub-optimal solution, it can still yield a comparable result with the optimal solution as in Figure 8, except for the very stringent requirement $\left(I_{t h}=1 / 2 \sigma^{2}\right)$. The introduction of windowing, Tukey win dow in this case, has significantly improved the system performance. As Tukey is very effective in reducing out-of-band interference, CR's sub-carriers far from PU's band will have negligible effect. Therefore, optimal algo-

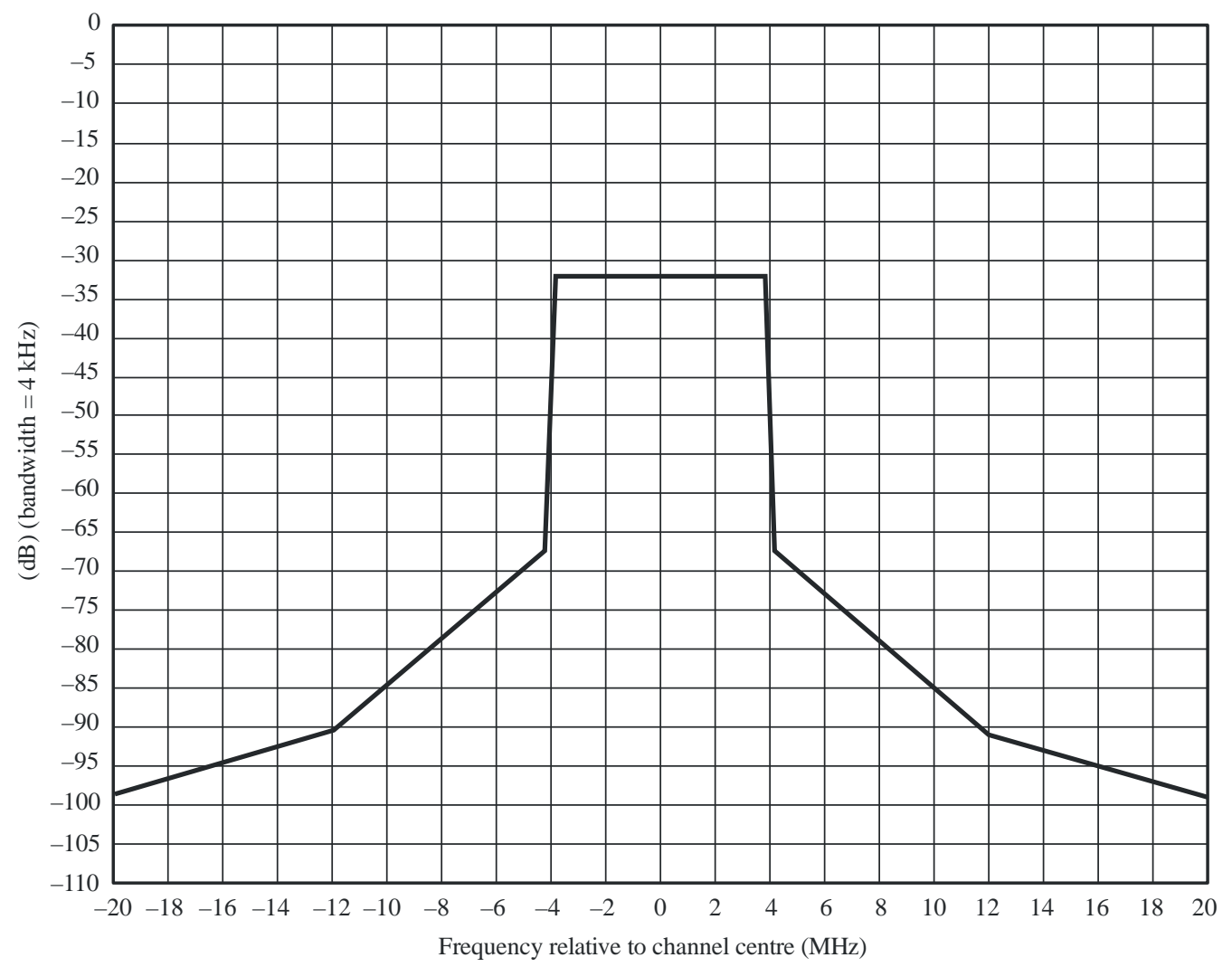

Figure 6. Emission mask of 8 MHz DVB-T transmitter. 
Table 1. Breakpoints for 8 MHz DVB-T systems.

\begin{tabular}{cc}
\hline Frequency relative to centre of the $8 \mathrm{MHz}$ channel & Relative level in $4 \mathrm{kHz}$ measurement bandwidth $(\mathrm{dB})$ \\
\hline-20 & -99 \\
-12 & -91 \\
-4.2 & -67.8 \\
-3.81 & -32.8 \\
3.81 & -32.8 \\
4.2 & -67.8 \\
12 & -91 \\
20 & -99 \\
\hline
\end{tabular}

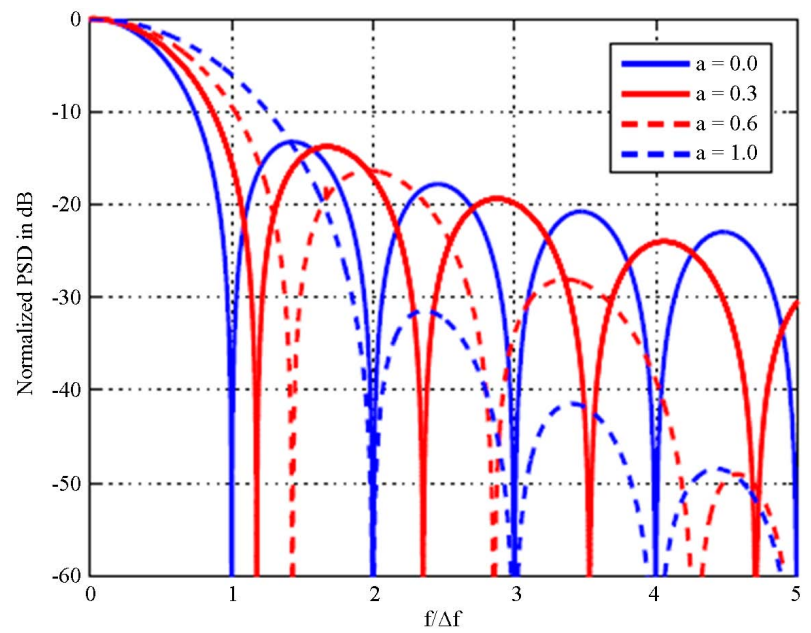

Figure 7. PSD of Tukey window with different roll-off factors.

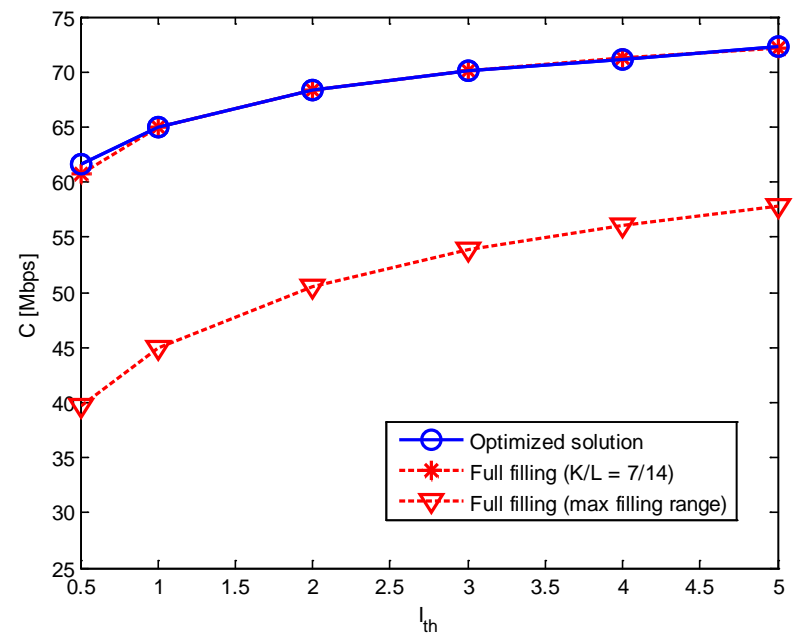

Figure 8. Channel capacity

rithm only plays its role to sub-carriers closed to PU's band. As a result it is not much different in terms of channel capacity when comparing optimal and sub-optimal PFR algorithms.

For comparison purpose, we provide the optimized power allocation solution in Figures 9, 10. With the si-

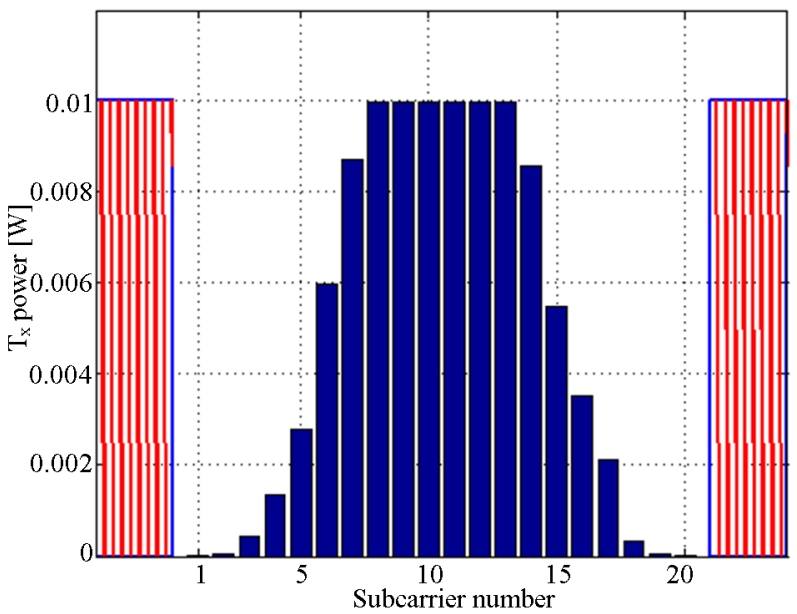

Figure 9. Optimized power allocation $\left(I_{t h}=1 \sigma^{2}\right)$.

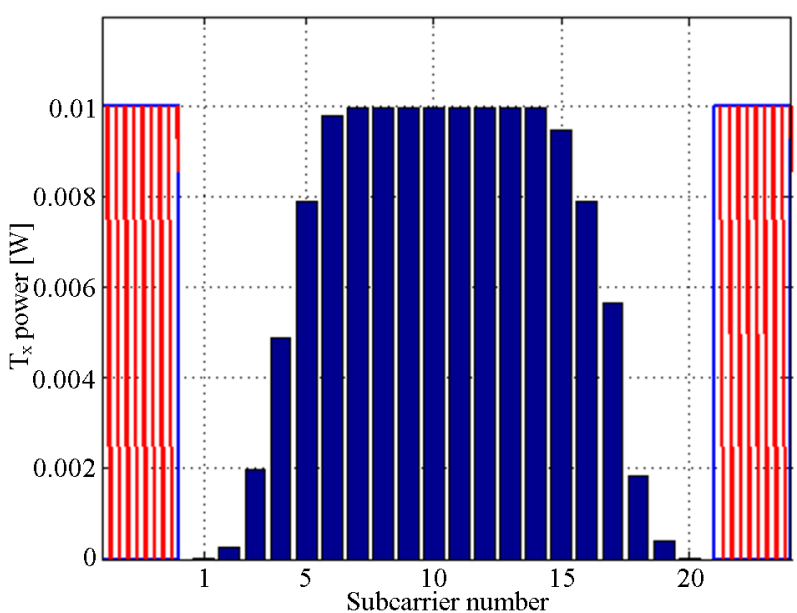

Figure 10. Optimized power allocation $\left(I_{t h}=5 \sigma^{2}\right)$.

mulation setting for PFR in this paper, filling range is set to sub-carrires from 7 to 14 , the simulation results are given in Figures 11, 12. When the requirements are quite stiff, the difference between PFR and optimum solution in power allocation pattern is quite obvious as in Figures 9,11 . When the requirement is less stringent, as in Figures 10, 12, the power allocation patterns of PFR and the optimum one are quite similar. 


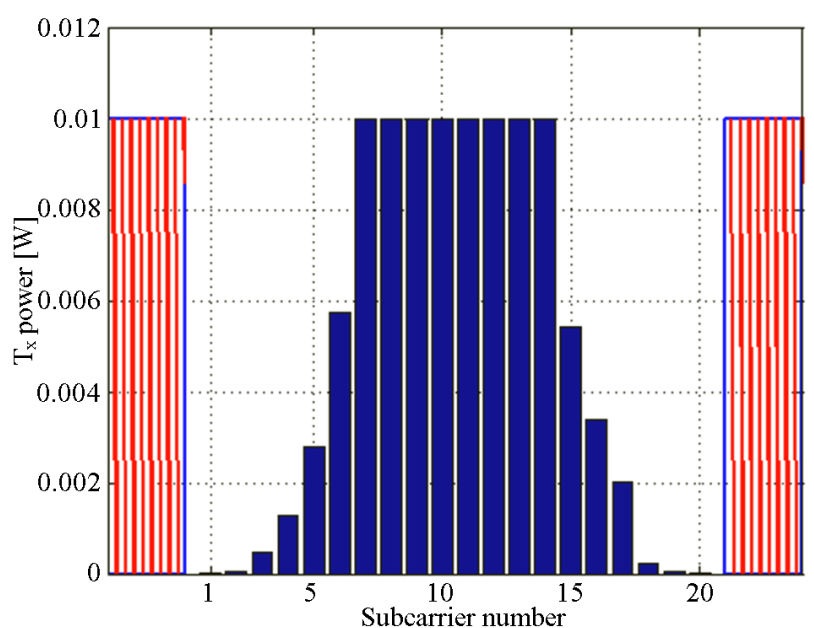

Figure 11. PFR's power allocation $\left(I_{t h}=1 \sigma^{2}\right)$.

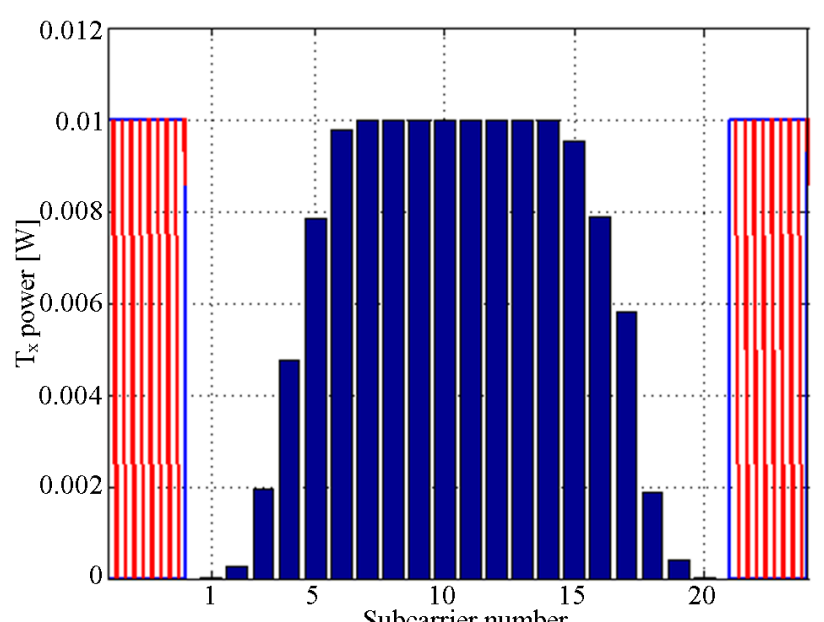

Figure 12. PFR's power allocation $\left(I_{t h}=5 \sigma^{2}\right)$.

We now turn to the power allocation of MFR algorithm.

As the sub-carriers adjacent to PU's bands cause the most interference to PU, even in the optimal solution, the power allocated to those sub-carriers are quite low indeed. Therefore, we can shut down those sub-carriers to reduce the computation complexity with small trade-off in the system throughput.

The MFR presents a light weight solution to the problem. It tries to allocate as much power as possible for suitable subcarrier, depending on the channel condition. Figures 13, 14 show different power allocation for a transmission in different conditions.

Depending on the channel at the moment of transmision, the power allocation may not have symmetry form, as clearly shown in Figure 14. However, when we view the average power allocation to all sub-carriers in the long term, we will have a perfect symmetrical form, as in Figure 15.

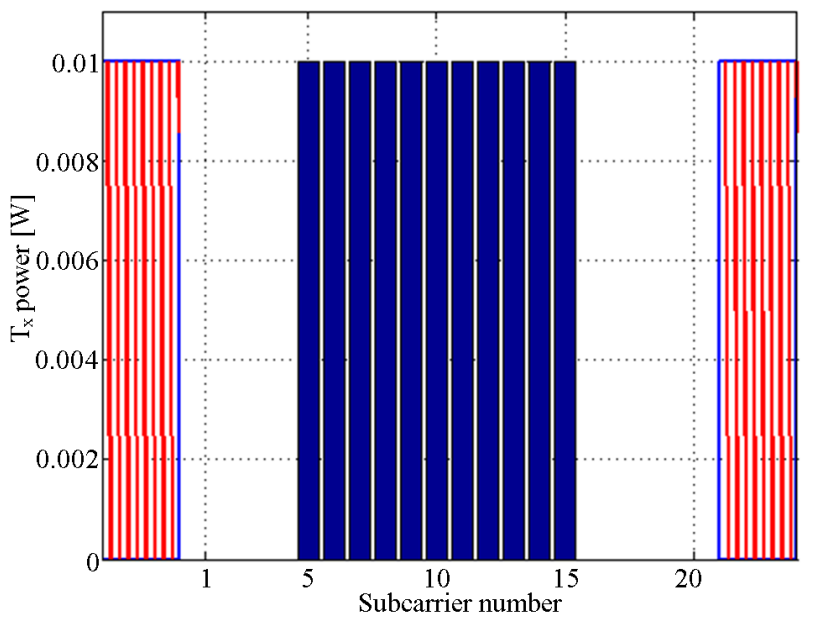

Figure 13. MFR power allocation (one snapshot, $I_{t h}=2 \sigma^{2}$ ).

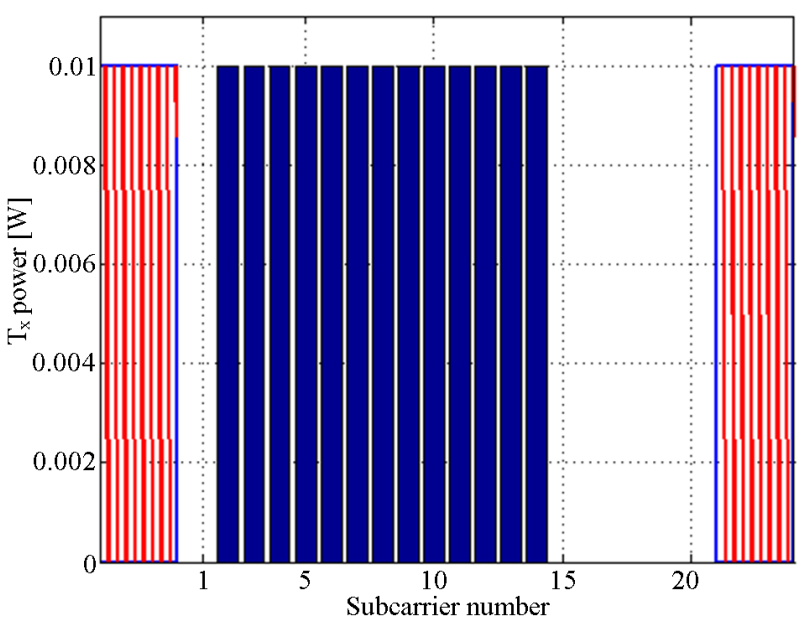

Figure 14. MFR power allocation (one snapshot, $\boldsymbol{I}_{t h}=5 \sigma^{2}$ ).

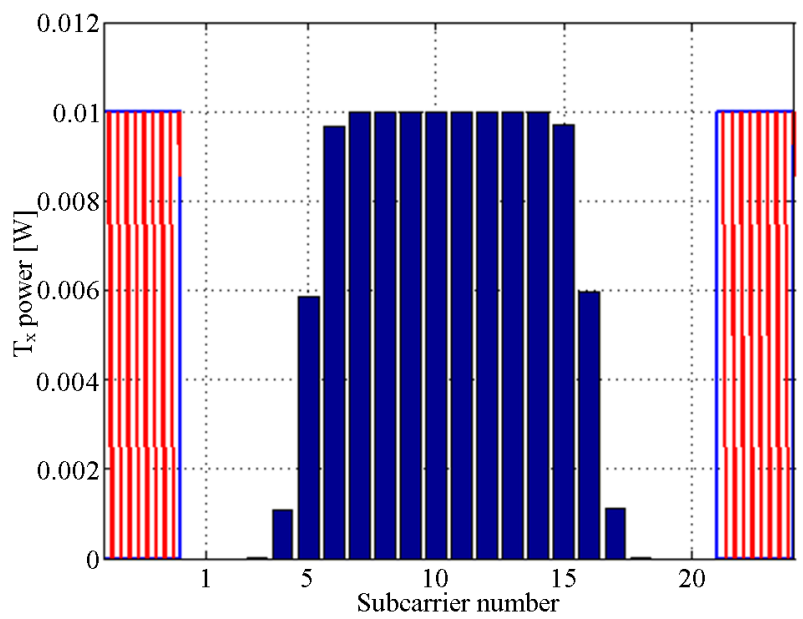

Figure 15. MFR's average power allocation $\left(I_{t h}=2 \sigma^{2}\right)$.

\section{Conclusions}

In this paper, we have introduced a new class of suboptimal power loading algorithm, called full-filling algo- 
rithm, to CR systems. Two sub-optimal solutions are proposed, MFR and PFR. Through extensive numerical analysis, we have shown the effectiveness of the proposed full-filling algorithm.

One of the striking advantages of full-filling algorithm is its low computational complexity. The proposed algorithm has significantly reduced the number of variables in the optimization problem. This directly leads to: simpler implementation, faster conversion rate, saving CR's computation power while achieving practically the same transmission rate.

The MFR algorithm proves to be a very promising proposal in term of low complexity. Beside simplicity, one of its shortcomings is that it does not take into account 2 adjacent sub-carriers to the filling range. In MFR, those two sub-carriers are set to 0 . We believe that allocating small power to those 2 sub-carriers can improve the CR's throughput with non-significant additional computation.

\section{REFERENCES}

[1] G. Bansal, M. J. Hossain and V. K. Bhargava, "Optimal and Suboptimal Power Allocation Schemes for OFDMBased Cognitive Radio Systems," IEEE Transactions on Wireless Communications, Vol. 7, No. 11, 2008, pp. 4710-4718. http://dx.doi.org/10.1109/T-WC.2008.07091

[2] "Adaptive Power Loading for OFDM-Based Cognitive Radio Systems," IEEE International Conference on Communications, ICC'07, Vol. 10, No. 9, 2007, pp. 51375142.

[3] G. Bansal, O. Duval and F. Gagnon, "Joint Overlay and Underlay Power Allocation Scheme for OFDM-Based Cognitive Radio Systems," Vehicular Technology Conference (VTC 2010-Spring), 2010 IEEE 71st, 16-19 May 2010, pp. 1-5.
[4] G. Bansal, M. J. Hossain and V. K. Bhargava, “Adaptive Power Loading for OFDM-Based Cognitive Radio Systems with Statistical Interference Constraint," IEEE Transactions on Wireless Communications, Vol. 10, No. 9, 2011, pp. 2786-2791. http://dx.doi.org/10.1109/TWC.2011.072011.100397

[5] T. Weiss, J. Hillenbrand, A. Krohn and F. K. Jondral, "Mutual Interference in OFDM-Based Spectrum Pooling Systems," Vehicular Technology Conference, 2004. VTC 2004-Spring. 2004 IEEE 59th, Vol. 4, 2004, pp. 18731877.

[6] H. A. Mahmoud and H. Arslan, "Sidelobes Suppression in OFDM-Based Spectrum Sharing Systems Using Adaptive Symbol Transition,” IEEE Communications Letters, Vol. 12, No. 2, 2008, pp. 133-135. http://dx.doi.org/10.1109/LCOMM.2008.071729

[7] Y. Zhang and C. Leung, "An Efficient Power-Loading Scheme for OFDM Based Cognitive Radio Systems," IEEE Transactions on Vehicular Technology, Vol. 59, No. 4, 2010, pp. 1858-1864. http://dx.doi.org/10.1109/TVT.2009.2039154

[8] T. Le Van, H. Nguyen Thanh, H. Dinh Chi and K. Nguyen Viet, "Impact of Windowing on Power Allocation in Cognitive Radio System,” The 4th International Conference on Communications and Electronics (ICCE) 2012, Hue, 1-3 August 2012, pp. 212-216.

[9] IEEE, “Standard for Information Technology Telecommunications and Information Exchange between Systems Local and Metropolitan Area Networks Specific Requirements Part 11: Wireless LAN Medium Access Control (MAC) and Physical Layer (PHY) Specifications,” Std., 2007.

[10] J. H. Rainer, "Applications of the Fourier Transform to the Processing of Vibration Signals,” Tech. Rep., 1986.

[11] ITU-R Recommendation SM.1541-4, 2011 